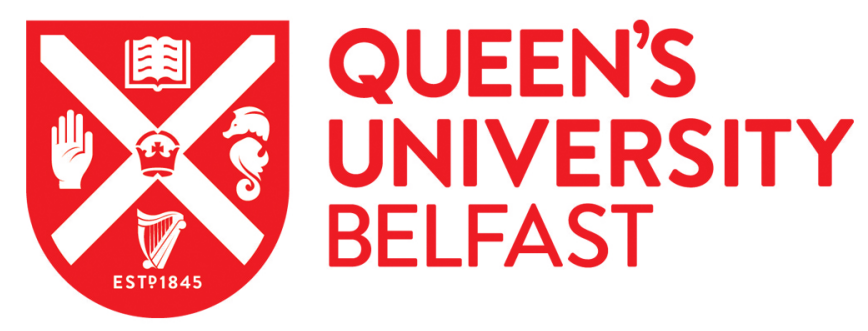

\title{
DTA-PUF: Dynamic Timing-aware Physical Unclonable Function for Resource-constrained Devices
}

Tsiokanos, I., Miskelly, J., Gu, C., O'Neill, M., \& Karakonstantis, G. (2021). DTA-PUF: Dynamic Timing-aware Physical Unclonable Function for Resource-constrained Devices. ACM Journal on Emerging Technologies in Computing , 17(3), [32]. https://doi.org/10.1145/3434281

Published in:

ACM Journal on Emerging Technologies in Computing

Document Version:

Peer reviewed version

Queen's University Belfast - Research Portal:

Link to publication record in Queen's University Belfast Research Portal

Publisher rights

Copyright 2020 The author. This work is made available online in accordance with the publisher's policies. Please refer to any applicable terms of use of the publisher.

\section{General rights}

Copyright for the publications made accessible via the Queen's University Belfast Research Portal is retained by the author(s) and / or other copyright owners and it is a condition of accessing these publications that users recognise and abide by the legal requirements associated with these rights.

Take down policy

The Research Portal is Queen's institutional repository that provides access to Queen's research output. Every effort has been made to ensure that content in the Research Portal does not infringe any person's rights, or applicable UK laws. If you discover content in the Research Portal that you believe breaches copyright or violates any law, please contact openaccess@qub.ac.uk. 


\section{DTA-PUF: Dynamic Timing Aware Physical Unclonable Function for Resource Constrained Devices}

IOANNIS TSIOKANOS, Institute of Electronics, Communications and Information Technology (ECIT), Queen's University Belfast, UK

JACK MISKELLY, Institute of Electronics, Communications and Information Technology (ECIT), Queen's University Belfast, UK

CHONGYAN GU, Institute of Electronics, Communications and Information Technology (ECIT), Queen's University Belfast, UK

MAIRE O'NEILL, Institute of Electronics, Communications and Information Technology (ECIT), Queen's University Belfast, UK

GEORGIOS KARAKONSTANTIS, Institute of Electronics, Communications and Information Technology (ECIT), Queen's University Belfast, UK

In recent years, physical unclonable functions (PUFs) have gained a lot of attention as mechanisms for hardware-rooted device authentication. While the majority of the previously proposed PUFs derive entropy using dedicated circuitry, software PUFs achieve this from existing circuitry in a system. Such software derived designs are highly desirable for low power embedded systems as they require no hardware overhead. However, these software PUFs induce considerable processing overheads that hinder their adoption in resource constrained devices. In this paper, we propose DTA-PUF, a novel, software PUF design that exploits the instruction- and data-dependent dynamic timing behaviour of pipelined cores to provide a reliable challengeresponse mechanism without requiring any extra hardware. DTA-PUF accepts sequences of instructions as an input challenge and produces an output response based on the manifested timing errors under specific overclocked settings. To lower the required processing effort, we systematically select instruction sequences that maximise error-rate. The application to a post-layout pipelined floating-point unit, which is implemented in 45 $\mathrm{nm}$ process technology, demonstrates the effectiveness and practicability of our PUF design. Finally, DTA-PUF requires up-to $50 \times$ fewer instructions than existing software processor PUF designs, limiting processing costs and resulting in up-to $26 \%$ power savings.

Additional Key Words and Phrases: device identification, dynamic timing analysis, FPU, hardware security, intrinsic processor PUF, IoT, pipeline, resource constrained devices, timing errors, software PUF

\section{INTRODUCTION}

The continuous scaling of transistor sizes and technology advances are driving the demand for low power portable devices [33, 58]. The International Data Corporation (IDC) [3] estimates that there will be 41.6 billion devices connected to the Internet, generating 79.4 zettabytes (ZB) of data in 2025 . Therefore, with the advent of Internet of Things (IoT) era, secure communication among computing devices is of prime importance [6, 22]. Traditional security methods (e.g., cryptography) [59,

Authors' addresses: Ioannis Tsiokanos, Institute of Electronics, Communications and Information Technology (ECIT), Queen’s University Belfast, , Belfast, UK, itsiokanos01@qub.ac.uk; Jack Miskelly, jmiskelly08@qub.ac.uk, Institute of Electronics, Communications and Information Technology (ECIT), Queen's University Belfast, , Belfast, UK; Chongyan Gu, Institute of Electronics, Communications and Information Technology (ECIT), Queen's University Belfast, , Belfast, UK, c.gu@qub.ac.uk; Maire O’Neill, Institute of Electronics, Communications and Information Technology (ECIT), Queen’s University Belfast, , Belfast, UK, m.oneill@ecit.qub.ac.uk; Georgios Karakonstantis, Institute of Electronics, Communications and Information Technology (ECIT), Queen’s University Belfast, , Belfast, UK, g.karakonstantis@qub.ac.uk.

(c) - -

XXXX-XXXX/-/-ART- \$-

$-/-$

, Vol. -, No. -, Article -. Publication date: - 
66] require intense computations and thus are undesirable for low power, resource constrained platforms. As such, research has been conducted into new lightweight and low overhead techniques for addressing hardware weaknesses $[41,49,70]$ as early as possible at design cycle [12, 22]. Physical Unclonable Function (PUF) [32] is one security primitive which has been proposed in this space.

\subsection{Physical Unclonable Function Overview}

PUFs are security primitives that derive entropy from low level manufacturing process variation in physical components. PUFs are a good candidate to address the security problems of resource constrained computing platforms, especially in regard to device identification (ID) and authentication. PUF signatures are produced by a challenge-response protocol: a unique response (output) is generated for a specific challenge (input). The form of the challenge and response is dependent on the design and desired properties. The main target of a PUF design is to generate sufficient entropy that each instance gives a unique response, while simultaneously ensuring the responses are reliable - within a range of acceptable limits - under different environmental conditions [60].

Typically a PUF is implemented on a discrete chip, added as a component to a complex circuit, or implemented on an FPGA. Such PUFs form the vast majority of PUF literature to date. The most well studied designs are the arbiter PUF $[29,40]$, which generates entropy from the difference in two identically placed delay paths with an arbiter at the end, and the Ring Oscillator (RO) PUF [42, 60], which uses the difference in frequency between two identical oscillators to generate the response. Such studies have introduced designs with desirable security properties, but which are not always suitable for use in the context of resource constrained and pre-existing devices due to the need to make circuit design changes and add hardware components to carry out the PUF function.

\subsection{Software PUFs}

A potential solution to these issues is software PUFs, the entropy of which is extracted from circuitry already present in a system without modification and purely by means of software. These PUFs have the advantage of having no hardware overhead and requiring no design changes to the device hardware. In addition, as they are software based, it is often possible to deploy such PUFs onto devices which are already in use. Several such designs have been proposed [35, 39, 40, 43, 57]. By their nature, each software PUF design relies on the characteristics of an existing component of hardware in the system. Hence, to allow these PUFs to be usable on a broad range of devices it is necessary to have viable designs using as wide a range of underlying hardware as possible.

The primary example of a software PUF is the SRAM PUF [35], in which the PUF response is formed from the power-on values of cells in SRAM memory modules. Although effective, SRAM PUFs require power cycling to access the PUF response which is not practical in all systems. Designs for software memory PUFs based on other memory technologies, such as DRAM and NAND flash memory, have also been proposed $[10,24,55]$ though like the SRAM PUF specific conditions are required for the PUF to be viable in a given system. For instance, not every electronic device is equipped with DRAM and flash memories.

In an attempt to widen the body of devices that can viably use a software PUF, the concept of PUFs deriving entropy from a processor itself has been explored [39, 40, 43, 57]. Such designs leverage processor delays, which are prone to variations, to derive the PUF challenge-response mechanism. Although effective, and in some cases relatively lightweight, the majority of these designs are not software PUFs and require additional hardware to achieve the PUF challenge response mechanism [39, 40,57]. The only existing software processor PUF [43] may generate responses without the need of extra circuitry, but it requires on board precise high speed pulse generators. Particularly, it requires the characterization of each instruction for various clock reduction levels in order to achieve a sufficient number of response bits. This complicates the 
challenge-response mechanism, which consumes a significant amount of time/power to produce unique outputs.

\subsection{Contributions and Outline}

In this paper, we introduce DTA-PUF ${ }^{1}$, a software PUF design that achieves unique and reliable responses with a minimal power overhead compared to existing designs. By leveraging timing errors of structures inherent to microprocessors instead of building additional circuit/logic, we propose a low power PUF architecture. The basic principle of the proposed DTA-PUF lies in the systematic exploitation of the dynamic timing behaviour of logic, which has never been fully exploited, to minimise processing effort. The proposed PUF design is derived from ubiquitous circuitry and hence can be employed to many platforms - especially to those requiring low power computations such as IoT devices. The main contributions of our work can be summarized as follows:

- We develop a novel, processor based, software PUF design leveraging the instruction- and data-dependent timing behaviour of pipelined cores. DTA-PUF derives entropy from the inherent complex manifestation of timing errors under carefully selected overclocked settings. Revealing such properties require pre-fabrication simulations, which we perform at one of the most accurate phases before the actual manufacturing, i.e., at post-layout timing analysis phase.

- We implement a design flow using commercial tools that allow us to systematically select instruction sequences that maximize timing error rates in any target pipelined core. DTA-PUF requires considerably smaller size of input challenge than comparable designs to provide the challenge-respone mechanism, limiting required processing efforts and leading to power savings.

- We demonstrate the concept on a pipelined, out-of-order, IEEE-754 compliant [1] floatingpoint unit (FPU) implemented in $45 \mathrm{~nm}$ process technology. The generated PUF responses are evaluated with regard to several statistical quality metrics including uniqueness, min-entropy and reliability. Our results show high values of uniqueness, min-entropy and reliability among the evaluated chips.

The paper is organized as follows: Section 2 outlines background information and limitations of existing processor based PUFs. Section 3 introduces the proposed PUF design, while Section 4 discusses the implementation of the proposed DTA-PUF. Section 5 presents the experimental results and Section 6 discusses related work. Conclusions are drawn in Section 7.

\section{BACKGROUND AND MOTIVATION}

In this section, we provide background information and discuss the most common processor based PUF designs, analysing the challenges that motivate our work.

\subsection{PUF Designs - Background}

PUFs are typically characterised by a set of input challenges and corresponding output responses. These are referred to as Challenge-Response Pairs (CRPs), the form of which vary depending on the PUF design. It is generally assumed for a PUF design being used for hardware ID that some agent, either the system provider or the user, will initially characterise the PUF to derive the full set of CRPs. It is further assumed that this information will be stored somewhere separate from the PUF. This is referred to as "enrolment". When the system needs to be verified a given challenge

${ }^{1}$ DTA-PUF: Dynamic Timing Aware Physical Unclonable Function 
will be sent to the PUF and the response will be compared to the expected value. This is referred to as "query".

Depending on the number of CRPs that can be generated from a single device, PUFs can be distinguished into weak and strong PUFs:

Strong PUFs are those PUFs which have a very large CRP space. Ideally, a PUF classed as such should have a CRP space which grows exponentially with the resources dedicated to the PUF. PUFs which have only a linearly increasing CRP space are not typically classed as strong PUFs. Strong PUFs are the more well studied class of PUF, but have seen minimal adoption. This is in part due to the proven vulnerability of many strong PUFs to modelling via machine learning (ML) [26, 54], though in recent years proposals to mitigate this issue have been made [44].

Weak PUFs are those PUFs which have a relatively small CRP space - in many cases only a single CRP as in the case of SRAM PUFs [35]. Despite being labelled as "weak", these PUFs have seen much more usage in practice as they generally have quite a high cost for an adversary to model $[19,53]$. Typically, weak PUFs are jointly considered with cryptographic methods [65] (e.g., encryption) to compensate for CRP scarcity.

\subsection{Variability in Nanometer Circuits}

Several PUFs leverage intrinsic timing variations of circuits to provide secure protocols $[39,40$, $43,57]$. In fact, the microelectronic substrate, on which modern circuits are built, is increasingly prone in variability. Most profound is the variation in circuit parameters of the manufactured chips (1) within die, (2) die-to-die and (3) over time.

Within-die (intra-die) variations account for the the variations that arise between different devices and interconnects that reside within the same chip. There are different sources of these variations (e.g., process, voltage, temperature and environmental factors) and may result up-to $50 \%$ frequency fluctuations [31].

Die-to-die (inter-die) variations refer to the variations that arise between different chips in the same wafer or different wafers. Die-to-die variations are mostly design independent and are mainly related to equipment properties (e.g., wafer placement, manufacturing lithography).

Over time variations. Wires and transistors in integrated circuits suffer substantial wear-out, leading to power and performance changes over time of usage. One of the main sources of such dynamic variations is the phenomenon of device aging, which became much more troublesome for the sub-45nm nodes [7].

Such variations affect the point of first failure [17] and thus the safe clock frequency between different chips or different cores within the same chip. To mitigate such phenomenon, designers apply a global frequency constraint that forces all the manufactured chips to operate at different frequency groups [51] depending on the minimum frequency achieved in their cores. In Section 5.2 , we use this performance or speed grouping of chips to represent delay variations effects.

\subsection{Processor Based PUFs}

Those entirely software based, processor PUFs proposed to date accept instructions as input challenge and take advantage of the fact that the point of failure of a given instruction and path delays vary from chip to chip to generate the output response.

To better illustrate how these PUFs work, let us assume a synchronous processor consisting of a set of $N$ combinatorial paths $P=\left\{P_{1}, P_{2}, \ldots, P_{N}\right\}$, which are characterized by their delays $D\left(P_{i}\right)^{2}$ for $i=1,2, \ldots, N$. As in any synchronous design, the conventional static timing analysis (STA) evaluates the longest timing path across all $S$ pipeline stages and determines the the clock period $(C l k P)$ at

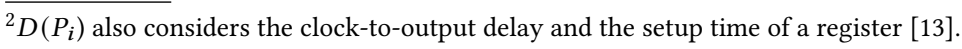

, Vol. -, No. -, Article -. Publication date: -. 


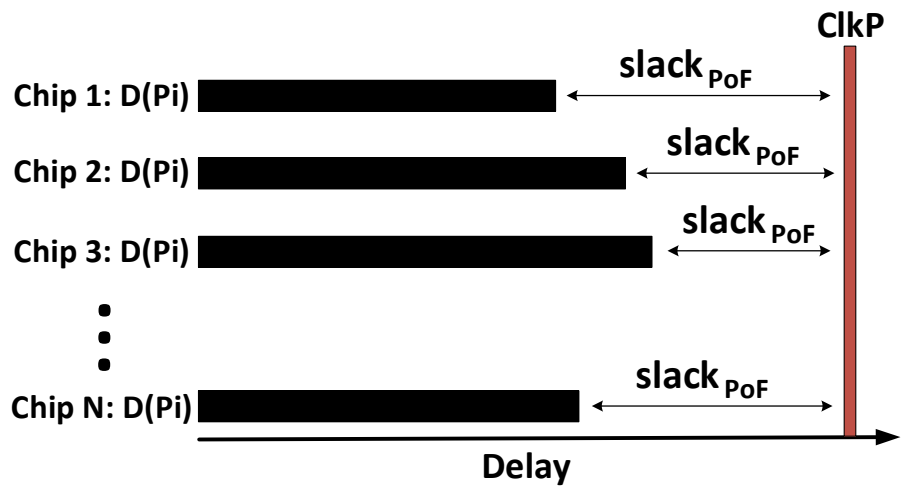

Fig. 1. Delay and point of failure (PoF) variations of the same timing path across variable chips.

design time, such as:

$$
C l k P \geqslant \max _{\mathbf{s}=1 \ldots . . .}\left\{\max _{\mathbf{p} \in \mathbf{P}^{\mathbf{s}}}\{D(p)\}\right\}=\max _{\mathbf{p} \in \mathbf{P}}\{D(p)\},
$$

where $P^{s}$ is the set of paths of pipeline stage $s(s=1,2, \ldots, S)$. During circuit operation, the executed instruction activates a path $P_{i}$ that has a positive timing slack, slack ${ }_{P o F}=C l k P-D\left(P_{i}\right)$, until the point of failure $(\mathrm{PoF})$. Under any clock period reduction, also known as overclocking, more than slack $_{P o F}$, the activated path $P_{i}$ will fail since $D\left(P_{i}\right)>C l k P$, leading to a setup timing error [13] Figure 1 provides an example where due to variations the same path $P_{i}$ has variable delay $\left(D\left(P_{i}\right)\right)$ and PoF $\left(\right.$ slack $\left._{P o F}\right)$ in different chips. Based on this figure, a processor PUF takes an instruction $I_{i}$ as input challenge $c h_{i}$, which sensitizes $P_{i}$, and generates an output response res $s_{i}$ exploiting variations in $D\left(P_{i}\right)$ and slack $_{P o F}$.

2.3.1 Non-software Processor Based PUFs. The majority of the existing processor PUFs extract entropy from path delay variations by introducing new circuitry to the processor for the purposes of response generation and extraction. In some cases, this is simply the addition of arbiters to measure existing delay paths [40], in others custom cores [8], and in others signal generation circuitry [5] [72] [69]. Such approaches require design changes to already complex circuits and incur a cost in terms of hardware resources, power budget, or both. These drawbacks limit their adoption in industry, especially in low power platforms. A full discussion of the various processor derived designs proposed to date can be found in Section 6.

2.3.2 Software Processor Based PUFs. In an effort to trim down the overheads induced by nonsoftware PUFs, a software processor PUF [43] has been proposed. To the best knowledge of the authors this is the only fully software based processor derived PUF design proposed to date. In this design, the response bits are generated by exploiting the fact that a given instruction fails under different frequency points across different chips. As such, it requires no extra circuitry or design changes to extract responses so long as the clock frequency can be controlled to induce the necessary instruction failures. Although effective, such an approach requires precise overclocking to a wide range of values and a high number of repeated instruction calls to generate just two bits of a response. Further, the overall CRP space is limited by the resolution of the clock. Each distinct clock period can generate only two bits per unique instruction, requiring a considerable number of instructions executed at different overclocking levels to generate an adequate output response (e.g., 128-bit response). While subsequent studies have tried to address these issues via added custom instruction logic [8], we demonstrate in this work that it is possible to generate PUF 


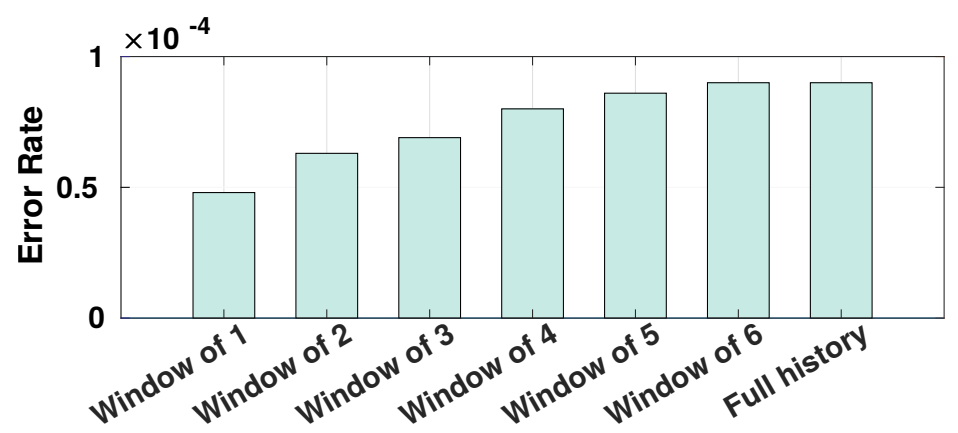

Fig. 2. Impact of instruction execution history on errors.

responses quickly and with much lower power consumption, while retaining a fully software based design. This is achieved by targeting pipeline cores and through the careful selection of instruction sequences as input challenges.

\subsection{Dynamic Timing Behaviour \& Instruction Execution History}

The existing software processor PUF [43] exploits the data-dependent path activation [28] to generate unique responses. However, it neglects the impact of instruction execution history (i.e., type and order of instructions within a pipeline at any instant) on the dynamic timing behaviour of computational paths. Instructions that are executed concurrently (i.e., they share the same hardware circuitry in a time-sharing fashion) may affect the possibility of timing errors because these instructions share control signals and execution stages, affecting the state of the forwarding logic, and thereby place great demand on circuit timing deadlines [61]. In a previous study [34], it was shown that instruction sequences (ISQs) have a significant impact on timing error rates, but they have not indicated how many instructions within a sequence affect this dynamic timing behaviour. Intuitively, all those instructions that precede an instruction in the pipeline may have an effect on the timing error behaviour of this instruction. To investigate this, we extract real floating-point instructions executing on an ARM A7 board. To achieve this, we extend an opensource profiling tool [46] and extract a trace of $1 M$ floating-point ISQs from the $b t$ program of NAS benchmark suite [11]. Then, we run post-layout gate-level simulation (see Section 4.2) based on this trace of an FPU, the details of which will be discussed later (see Section 5.1). We simulate this trace in windows of increasing number of concurrently executed instructions under $15 \%$ clock reduction. Specifically, we start simulation with a window size of 1 instruction and increase it up-to the pipeline depth, which is 6 stages in the FPU under test. Each experiment records the timing error rate (ER), defined as:

$$
E R=\frac{\text { Faulty ISQs }}{\text { Total ISQs }}
$$

Figure 2 indicates that sequences consisting of 6 instructions have exactly the same ER when running the full trace (full history) through simulation. By contrast, a window size of 1 instruction leads to $\sim 46.6 \%$ lower ER when compared to the 6 instruction window. From these findings, we conclude that all the pipelined instructions preceding the currently executed instruction may trigger a timing error in that instruction.

Taking into consideration the above, we propose DTA-PUF: a processor PUF design which overcomes the limited response bit generation and minimises processing/power overheads by fully 

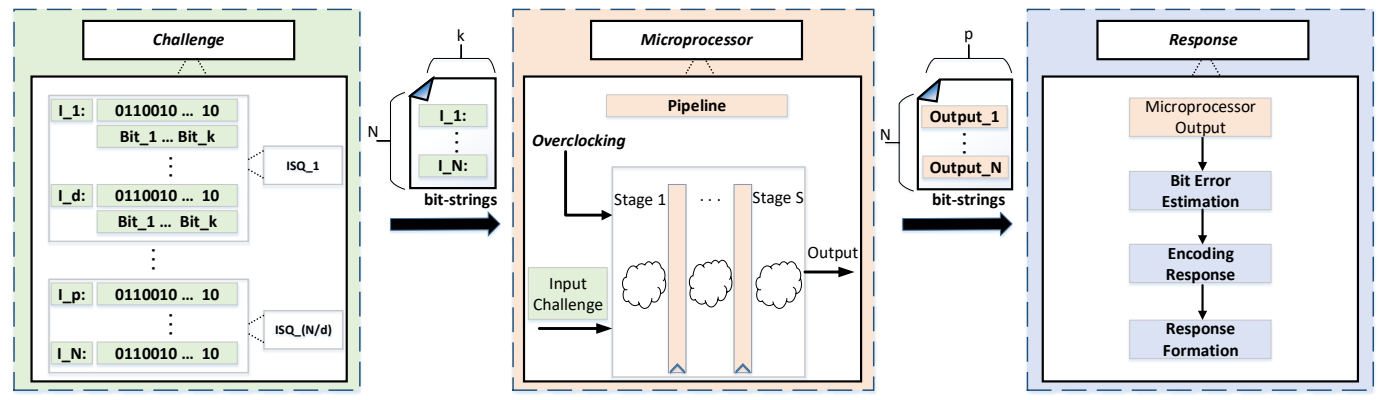

Fig. 3. Challenge response mechanism of DTA-PUF.

exploiting all the factors that affect error rate in pipelined units. The design is described in detail in the following section.

\section{PROPOSED SOFTWARE PUF}

DTA-PUF is a novel software PUF that extracts entropy with minimal resource usage by fully exploiting the dynamic timing behaviour of microprocessor circuits. DTA-PUF aims to jointly consider the inherent complexity of timing error manifestation with the intrinsic chip variations, proposing a new approach that inseparably intertwines PUF performance with dynamic error behaviour of pipelined cores. Our PUF implementation and evaluation rely on detailed post-layout gate-level simulation which is one of the most accurate, pre-fabrication steps of the standard ASIC flow used in industry [52]. Figure 3 shows the proposed PUF design for generating the challenge-response mechanism.

\subsection{Challenge Procedure}

In this work, the input challenge consists of a set of $N$ instructions $I=\left\{I_{-} 1, \ldots, I_{-} N\right\}$. An instruction is composed of a string of $k$ bits. It is important to note that these instructions are carefully selected to activate timing critical paths that maximise timing errors under a potential clock reduction. To this end, we use microarchitecture-aware timing information extracted at the design cycle (see Design Phase of Figure 4). As already explained, instruction execution history plays an important role in timing error manifestation. To create an execution history-aware challenge, while considering the data-dependent error occurrence, we split $I$ into sequences $I S Q \_n$ consisting of $d$ instructions such that $\cup_{n=1}^{N / d} I S Q \_n=I$.

\subsection{Microprocessor Operation}

The generated challenge is then given as an input to the target microprocessor. This PUF accepts $I$ as a challenge and produces the count of the bit errors in the output of the microprocessor as the response. Since $I$ consists of $N$ instructions (or $N / d$ ISQs), the microprocessor outputs a string consisting of $N \times p$ bits, where $\mathrm{p}$ depends on the architecture of the design under test. Timing errors are captured by operating the microprocessor pipeline at a reduced clock period, known as overclocking. We refer to the magnitude of this clock reduction as $\Delta T$. Note that $\Delta T$ should not exceed a certain clock period beyond which the microprocessor fails completely (we refer to as $T_{\text {fail }}$ ). In particular, $\Delta \mathrm{T}$ should be between $P o F$ and $T_{\text {fail }}$ such that $T_{\text {fail }}>\Delta T \geqslant P o F$. It is important to mention that such an overclocking technique is applied to target microprocessor when it operates as a PUF; during the normal operation, the nominal clock period is used (see Section 3.4). 


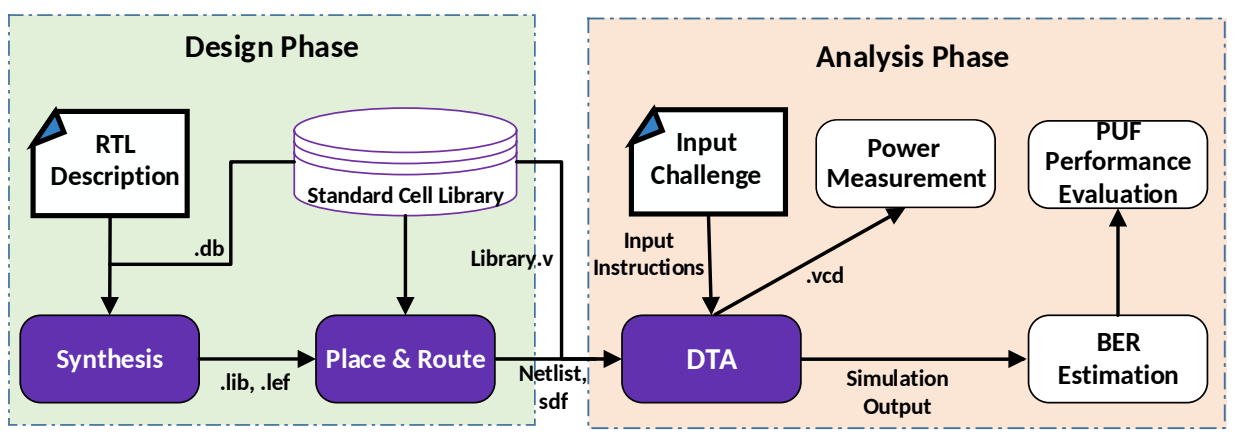

Fig. 4. Design and analysis phases of DTA-PUF.

\subsection{PUF Response}

The response is extracted from the output of the ISQs by counting the bit errors of the final instruction in each sequence. While in some cases multiple erroneous instructions occur within a sequence, we observed that the greatest entropy was achieved only in the final instruction of a sequence of $d$ instructions. Additionally, we observed that some bits had near zero entropy, and thus were not useful for constructing the response. This is explained in further detail in Section 5. The number of errors is encoded as a 6 bit binary value (the amount of bits needed to represent the possible error values of the 64 bit output minus the excluded bits). These values are concatenated to create a response of the desired length. For example, assume an $n=18$-bit response is required. Three ISQs are used which produce 1, 9, and 7 bit errors respectively. The corresponding binary responses would be 000001,001001 and 000111 . The resultant 18-bit response is the concatenation of these bit-strings, i.e. 000001001001000111.

\subsection{Microrchitectural Support}

The target microprocessor supports two modes: i) the normal, fully accurate mode where a set of instructions executed in the pipeline at the nominal clock period, and ii) the PUF mode where the microprocessor operates at a reduced clock period. To this end, we extend the instruction set of microprocessor by two special instructions PUFstart and PUFstop. PUFstart indicates that the microprocessor will explicitly operate as a PUF (PUF mode). Once the PUF response has been generated, the PUFstop instruction forwards the PUF response and switches the microprocessor to the normal operation mode. Since the PUF mode disrupts the microprocessor normal operation, there is an execution time overhead on programs executed in normal mode. However, as we will explain in Section 5.6, DTA-PUF's high bit generation speed limits such overhead.

\section{IMPLEMENTATION WORKFLOW}

The implementation workflow of DTA-PUF is depicted in Figure 4. The design phase is being executed only once, while the analysis phase runs for each challenge.

\subsection{Design Phase}

The first step of this phase is the Synthesis which is followed by the Place and Route steps. These steps are performed utilizing optimizations which aim to achieve maximum performance. The design phase outputs the following files:

i) A library verilog file which specifies the logic and the rise and fall times of the standard cells.

ii) A gate-level netlist which is stored in a verilog format (.v). This file consists of a list of the 
electronic components in the circuit and a list of nodes they are connected to.

iii) A standard delay format (SDF) file which describes the cell and interconnect delay. This file is obtained at the Place and Route step.

Design phase also generates a timing report that includes delays of the timing paths. This helps to create an input challenge set (see Section 3.1) that activates timing critical paths, leading to an increased number of timing errors at a reduced clock period.

\subsection{Analysis Phase}

Dynamic Timing Analysis (DTA). To enable characterization of the data-dependent path activation, we perform DTA using post-layout gate-level simulation. DTA identifies the actual timing margins of the target core at runtime by including path activation information (instruction type, operand values, pipeline sequence) that is unavailable during static timing analysis. The DTA tool uses as input the generated challenge (see Section 3), the outputs of the design phase and the set clock period. Providing that every set of instructions under nominal conditions and clock period produces an error-free output, we define this simulation output as $O_{\text {gold }}$. We estimate the number of timing failures by comparing $O_{\text {gold }}$ with the simulation output under the reduced clock period. Timing errors in the final instruction of each ISQ are then used to form an $n$-bit PUF response. Based on the output response, we utilize three quality metrics to evaluate the DTA-PUF performance: uniqueness, min-entropy and reliability.

Such an analysis phase also helps to extract a value change dump (VCD) file essential for power analysis. This file contains information about the switching activity and value changes occurred during the simulation.

\section{EVALUATION RESULTS}

In this section, we first present our experimental set-up. Second, we evaluate the efficacy of DTAPUF for generating unique and reliable responses. Third, we estimate any possible power gains by exploiting the high bit generation speed of DTA-PUF compared to an existing processor based software PUF design. Then, we present several use cases of DTA-PUF and lastly, we discuss our PUF vulnerability to potential attacks.

\subsection{Experimental Set-up}

For the case study of using DTA-PUF, we focus on arithmetic, floating-point operations since those are more prone to timing errors, as also reported by existing studies [15, 37, 43, 63]

We apply our approach to a 6 stage pipelined, out-of-order, IEEE-754 compatible [1] FPU that supports double precision operations. According to the IEEE-754 Standard, a floating-point number follows the representation $-1^{S} \times M \times 2^{E}$, where $S$ : sign, $\mathrm{E}$ : exponent and $M$ : mantissa. In a double precision FP number the most significant bit (MSB) indicates the sign, the next 11 bits represent the exponent and the mantissa consists of the last 52 bits. This unit is part of the mor $1 \mathrm{kx}$ MAROCCHINO pipeline, which is a single-core processor based on the OpenRISC-1000 instruction set architecture [4]. The FPU supports the following floating-point instructions: multiplication, division, addition and subtraction, integer-to-float and float-to-integer conversions. Figure 5 illustrates the microarchitecture of the targeted FPU, highlighting the floating-point addition/subtraction. At Stage 1, an Order Control Buffer and a Pre-Normalize block are implemented, which permits data dependencies detection and adjustment of the exponent and mantissa, respectively. Stage 2 is responsible for the pre-addition/subtraction alignment, while Stage 3 performs the necessary multiplexing and shifting of the operands. Mantissa addition and exponent update are performed at Stage 4; rounding occurs in the last two stages.

, Vol. -, No. -, Article -. Publication date: - 


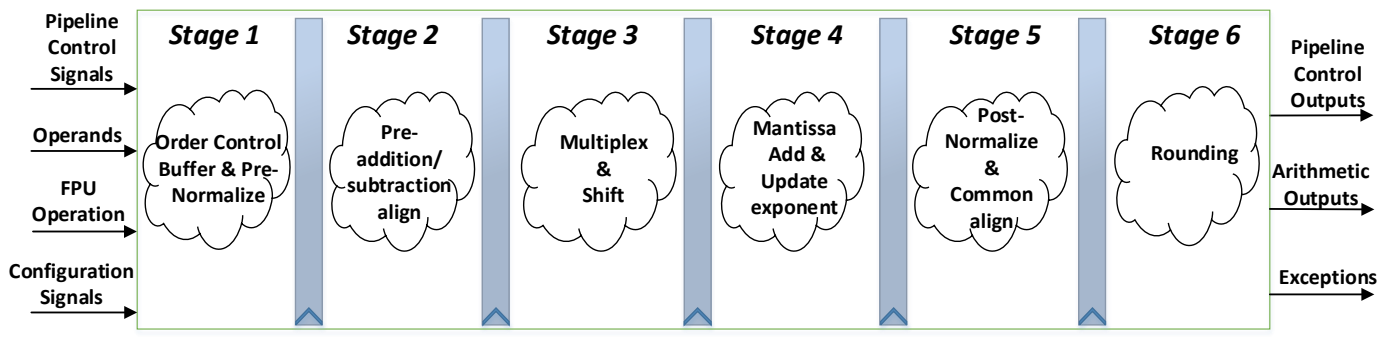

Fig. 5. Microarchitecture of the floating-point additions/subtraction related stages.

Based on OpenRISC 1000 instruction set architecture and IEEE-754 Standard, the input challenge of the PUF consists of $N$ instructions from $k=140$-bits each. We simulate these instruction in sequences consisting of $d=6$ instructions, where $d$ corresponds to the pipeline-depth of the FPU under test and thus to the maximum number of concurrently executed instructions in the pipeline. While simulation output of the FPU is composed of $p \times N$ bits, where $p=64$. $N$ strongly depends on the number of the bits in PUF response (see Section 5.E).

This FPU design is implemented using the typical corner of the CCS NanGate $45 \mathrm{~nm}$ library (@1.1V) [2]. For hardware Synthesis and Place and Route, we use the Design Compiler (version: N-2017.09-SP3) from Synopsys and Innovus (version: v16.13-s0451) from Cadence, respectively. For the power measurements, we invoke Voltus (version 16.2) from Cadence. DTA is performed using detailed post-layout gate-level simulation supported by ModelSim (version 10.7c) from Mentor Graphics. The maximum clock frequency achieved is $425 \mathrm{MHz}$, i.e. $\mathrm{ClkP}=2.35 \mathrm{~ns}$. For these experiments, we capture timing error by reducing $C l k P$ by $\Delta T=30 \%$. We refer to the reduced clock period as $C l k P_{\text {red }}=1.65 n$.

\subsection{Representing Delay Variability of Integrated Circuits}

Due to increased static and dynamic variations of nanometer circuits, the delay of chips after fabrication will be different (see Section 2). In fact, such a delay uncertainty is manifested in the form of core-to-core and chip-to-chip frequency variations. As explained in Equation 1, the clock frequency/period can be modeled as the maximum delay of all the timing paths in the chip under test. For example, in the Intel's 65nm 80-core chip (@1.2V) [31], the maximum core frequency is achieved at $7.3 \mathrm{GHz}$, whereas the minimum one is $5.7 \mathrm{GHz}$. Such a frequency fluctuation has been also observed between different chips [14, 20,31,38]. The most common technique to model this, is the so-called speed or frequency binning $[18,56]$, where different chips (that implement the same functionality) fall into separate speed/frequency bins.

Therefore, we sort the considered chips within 10 clock frequency bins depicted in Figure 6 . Following [16, 40, 68], we also assume that clock period variations in different chips follows a random/Gaussian distribution $N\left(\mu, \sigma^{2}\right)$ with $\sigma=0.08 n$ s and $\mu=C l k P_{\text {red }}=1.65 n s$. We vary the clock period between $-3 \sigma$ to $+3 \sigma$ from $C l k P_{\text {red }}$ and the clock period of each chip is estimated as follows:

$$
C l k_{-} C_{m}=C l k P_{\text {red }} \pm \text { Tvar }_{m}
$$

where $m(m \in[1,50])$ denotes the total number of considered chips and Tvar $_{m}$ ( Tvar $_{m} \in$ $[-3 \sigma,+3 \sigma])$ the deviation of $C l k_{-} C_{m}$ from $C l k P_{r e d}$. In other words, this clock stretching technique represents as a single factor the overall influence of potential worst-case delay variations between chips. Additionally, the levels of variation-induced delay fluctuations (i.e., $\sigma$ and vvar $_{m}$ ) used in these experiments are consistent with what have been reported in literature [14, 31, 39, 57]. 


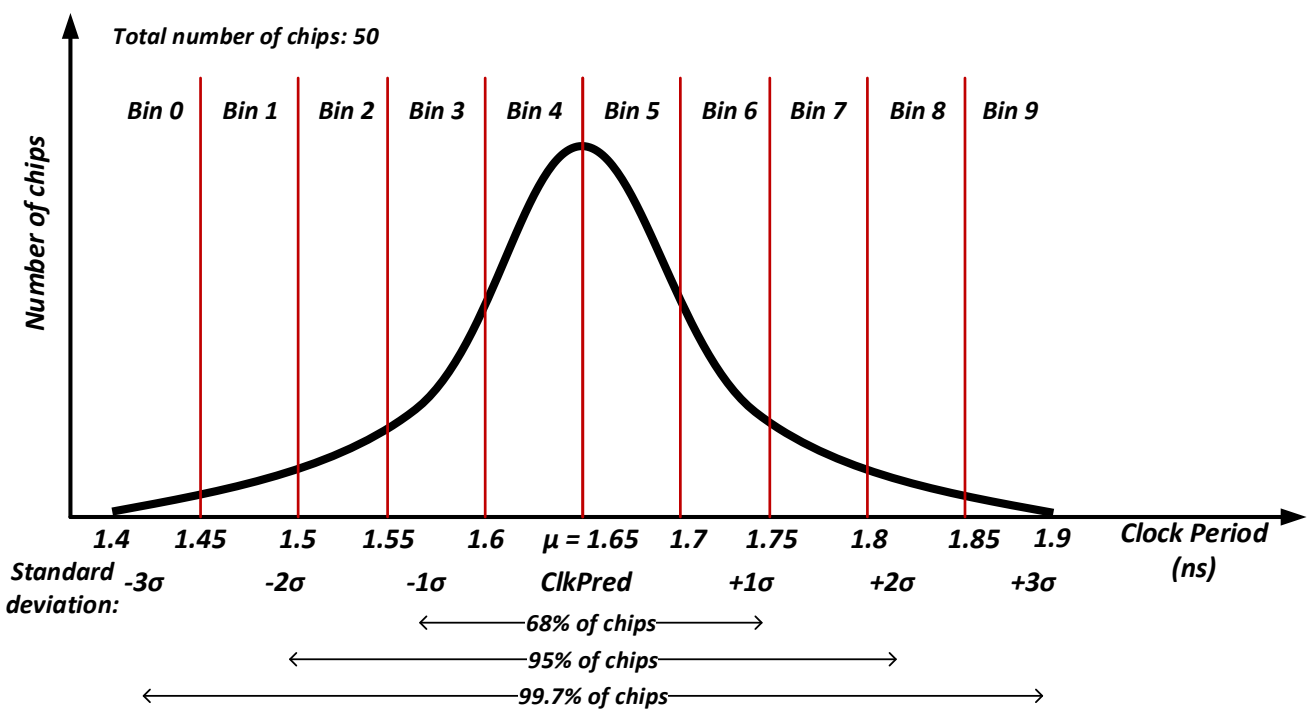

Fig. 6. Speed binning. Random/Gaussian distribution of clock period across the considered chips.

It is important to note that such a technique does not provide a perfectly accurate representation of real hardware, however, it sufficiently reflects the actual timing behaviour of circuits after fabrication as recently indicated $[37,64]$. Nonetheless, such a delay representation is based on detailed post-layout gate-level simulation which is among the final steps of the typical ASIC design flow used in industry [52]. In general, in the IoT regime where million of devices are connected to the Internet, there is a need to address hardware security challenges and validate the effectiveness of security protocols at design cycle, i.e. before the actual manufacturing of the chips.

Overall, we perform 50 simulations, representing $m=50$ different chips. Based on Gaussian distribution properties, we randomly select 34 chips (68\% of total chips) from bin 3 to bin 6 and 16 chips from the rest of the bins. The plots in Figure 7, Figure 8 and Figure 9 reflect this setting.

\subsection{Uniqueness}

In this subsection, we evaluate the effectiveness of our PUF in generating unique response to a given challenge in any two randomly selected chips in the overall population.

Uniqueness is calculated here by comparing the response of each chip to every other chip in the population as shown below, where $m$ is the number of PUFs in the population, $R n$ is the response of a given PUF instance, and $n$ is the number of response bits:

$$
U=\frac{2}{m(m-1)} \sum_{i=1}^{m-1} \sum_{j=i+1}^{m} \frac{H D\left(R_{i}, R_{j}\right)}{n} \times 100 \%
$$

Ideally, the uniqueness of all the possible CRPs will be a normal distribution centred around $50 \%$. As can be seen in Figure 7, the median uniqueness of this design is in the order of $26 \%$. Note that we use $|I|=9 M$ or $1.5 M$ ISQs as input challenge across $m=50$ chips/simulations to extract the uniqueness distribution depicted in Figure 7.a. Some ISQs show higher uniqueness than others across the entire population of devices and as such it is possible to be more selective when choosing which ISQs will be used as CRPs to improve the uniqueness value at the cost of reducing the CRP space. When such a technique is applied to select the best $90 \mathrm{~K}$ rather than $9 \mathrm{M}$ instructions as in 


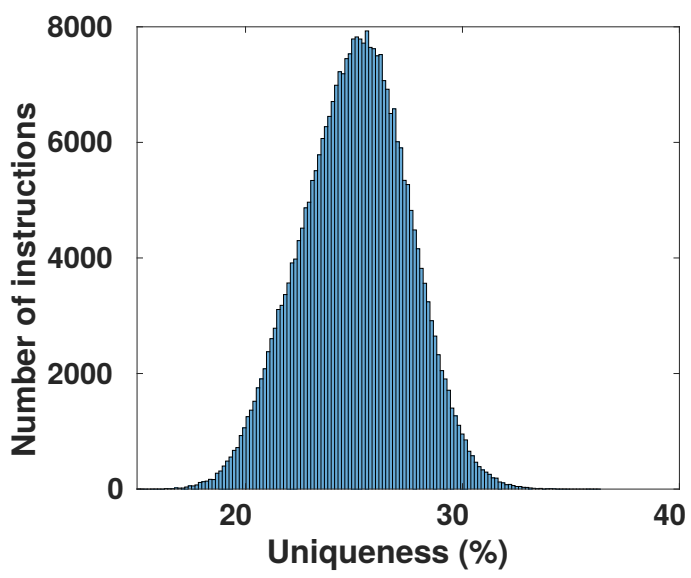

(a)

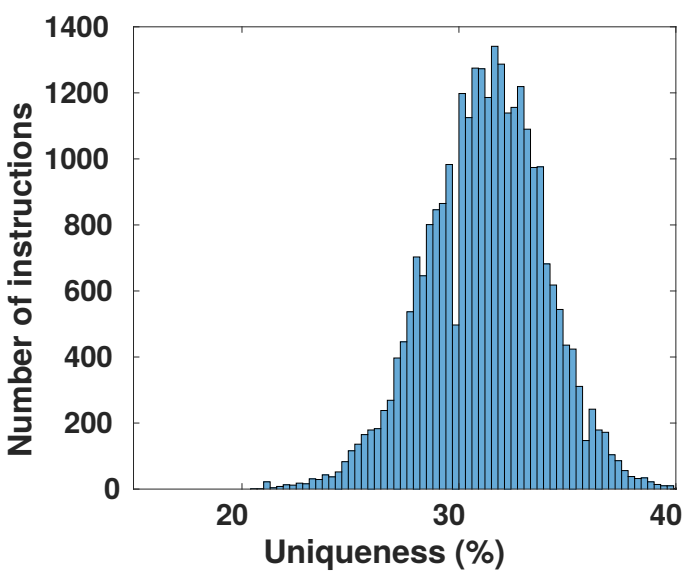

(b)

Fig. 7. Uniqueness distribution of the analysed CRPs using (a) $9 M$ instructions with no post-processing and (b) best-case selection of $90 \mathrm{~K}$ instructions with minimal processing effort.

the full experiments, the average uniqueness increases to $27.9 \%$. Additionally, if certain bits are excluded from the raw response and the resultant error rate encoded as a 6-bit response along with the previous technique, then, the average uniqueness further improves to $31.4 \%$; and it is likely that with further development this could be improved at the cost of increased complexity. The derivation of this 6 bit response is thoroughly explained in Section 5.4 below.

\subsection{Min-Entropy}

Min-Entropy $\left(H_{\min }\right)$ measures the worst-case entropy of the design, i.e. the minimum difference that will be seen between any two instances in the worst-case. The ideal value of $100 \%$ will be measured in a design in which any given bit being equal to 0 or 1 is equal probable. Min-Entropy is calculated as follows for an $n$ bit response measured across $m$ devices, where $P b_{\text {max }}$ denotes the maximum bit probability.

$$
\begin{gathered}
P b_{\text {max }}= \begin{cases}\frac{H W_{b}}{m} & H W_{b}>\frac{m}{2} \\
1-\frac{H W_{b}}{m} & H W_{b} \leq \frac{m}{2}\end{cases} \\
H_{\text {min }} b=-\log _{2}\left(P b_{\text {max }}\right) \\
H_{\text {min }}=\frac{1}{n} \sum_{b=1}^{n} H_{\text {min }} b
\end{gathered}
$$

An initial evaluation of $H_{\text {min }}$ was performed using the raw 64-bit output of every instruction in the instruction set for all devices, i.e. for $n=64$ and $m=50$, in order to evaluate if the raw output itself was usable as a PUF response. The encoding of bits into PUF responses in this case was kept as simple as possible, with the each bit of the response being a 0 if the bit was the expected output and a 1 if the bit was erroneous. The average $H_{\min }$ measured across this set of instructions was $11.68 \%$ which is very low. However, it was observed that there were distinct trends, when the bitwise Min-Entropy $\left(H_{\min } b\right)$ was averaged across the instruction set.

Firstly, the sign and exponent bits had near zero min entropy. This matches with what would be expected as the uppermost bits of a pipelined core are in general the least prone to error, meaning 


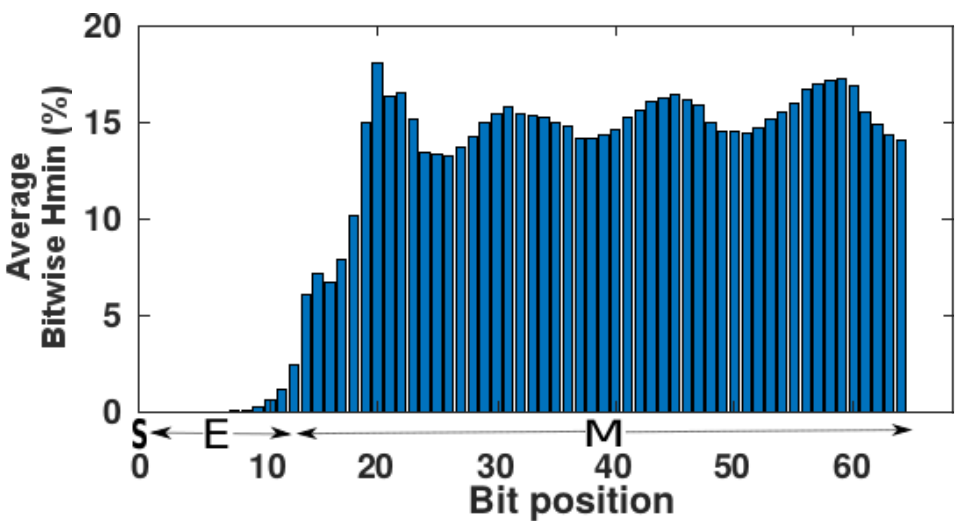

Fig. 8. Distribution of Min-Entropy across output bits. $\mathrm{X}$ axis depicts the sign (S) bit, the exponent (E) bits and the mantissa $(M)$ bits.

that these bits across almost all instructions were the expected value and hence produced a 0 in the PUF response. Further, even within the mantissa bits the first 10 bits were much lower in terms of average $H_{\min } b$; and while after this point the values remain relatively high, there are still distinct peaks and troughs. This can be clearly seen in Figure 8. From this, it can be implied that it is beneficial to exclude specific bits from the output when encoding the response as these bits will very rarely generate any errors, and hence will produce near identical response bits even across a relatively large number of challenges such as the $9 \mathrm{M}$ instructions evaluated here. Consequently, we select to filter out the low entropy bits and compare only the output of the bits that are likely to generate errors when forming the response. Specifically, the worst 33 bits in terms of $H_{\text {min }} b$ were excluded and the error rate of the remaining 31 bit output was encoded using 6 bits. Even then, the average $H_{\min } b$ is still only in the order of $15 \%$, however, this can be further improved by encoding the bit error rate as the response rather than the raw output, in which case the overall average $H_{\min }$ increases to $25.78 \%$. This combined with more selective choice of ISQs when forming challenges can also increase the uniqueness of the design to $31.4 \%$, as can be seen in Figure 7.b.

Each of these improvements in $H_{\text {min }}$ and uniqueness come at the cost of lowering the amount of bits generated per ISQ. With only the simplest post processing, 64 bits are generated per ISQ, while in the final scheme this is reduced to 6 . However, even with this reduction in throughput, the number of instructions required in comparison to existing fully software based processor derived PUFs is still greatly reduced with a corresponding reduction in the power consumption. This is explained in Section 5.6.

\subsection{Reliability}

As they exploit low level variations in the circuit fabrication, PUFs are often vulnerable to influence from environmental factors such as transistor aging or supply voltage fluctuations [7]. Reliability measures the amount by which the response of a given chip will vary under non-nominal environmental conditions. Reliability has been calculated by comparing a set of responses measured under varying conditions to a reference response, where $m$ is the number of measurements taken, Rref is the reference response, $R i$ is the response under varying environmental conditions, and $n$ is the number of response bits:

$$
\text { Rel }=\frac{1}{m} \sum_{i=1}^{m} \frac{H D\left(R_{r e f}, R_{i}\right)}{n} \times 100 \%
$$




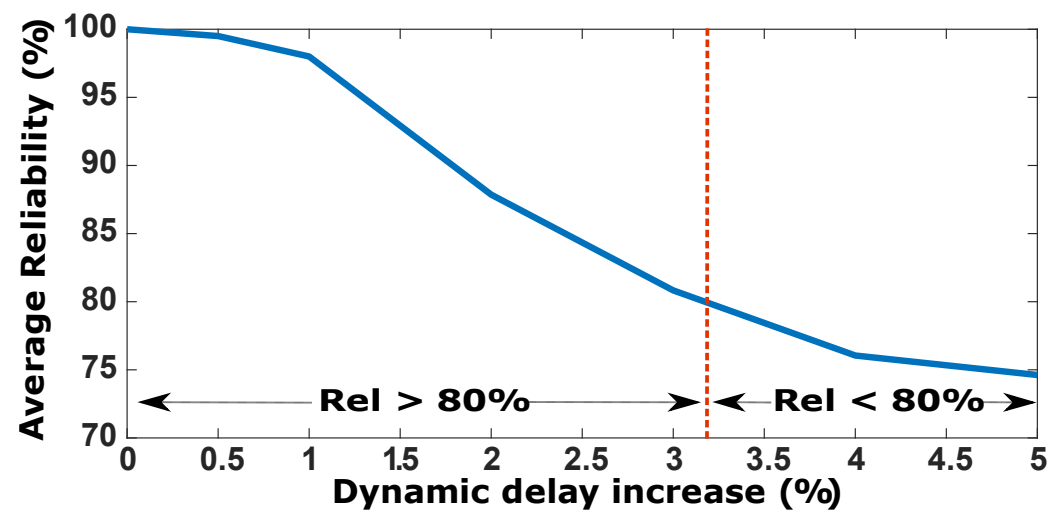

Fig. 9. Average reliability of response generation across different levels of environmental induced delay increase.

As our evaluation results are based on an accurate, post-layout gate-level simulation (see Figure 4), we have added an additional delay increase on top of the static delay variance, reflecting dynamic/over-time variations of the same chip. In order to provide a robust analysis of the likely PUF performance, we have measured the average reliability under increasingly high, environmentally induced variation up-to $5 \%[39,57]$.

As depicted in Figure 9, even under relatively large amounts of environmentally induced variance, the average reliability does not drop below $74 \%$. Further, so long as this variance can be controlled such that it does not exceed $3.2 \%$, the average reliability will remain above $80 \%$. This is insufficient for use as input to a cryptographic algorithm, but it suffices for the purpose of chip ID or IoT node ID. The three primary sources of such dynamic variance in this instance are temperature, supply voltage, and transistor-aging. In regards to temperature and supply voltage, we can mitigate the influence of these factors by enrolling the PUF initially at a variety of temperature-voltage combinations [50]. The current supply voltage and temperature can then be passed as supplementary data with the response, such that the PUF response can be compared with the expected response for the temperature and voltage which is closest to the current conditions. To account for transistor aging, it is possible to completely or partially re-enrol the PUF after a set period or to provide low cost, aging-aware mechanisms [7]. Re-enrolling our PUF induces an execution time overhead on the executed application, but as we explain in the next subsection (Section 5.6) the high-speed response generation keeps this overhead small. The circuit level, aging-aware technique presented in [7] explores approximate computing principles $[45,71]$ in the context of aging. Thus, it comes with an output quality loss that may be accepted by many applications [23, 30]. Specific methods to improve the reliability of this design are planned for future work, but are out of scope for this paper.

\subsection{Power Consumption, Execution Time and Output Response}

Using the simple concept explained in Section 3.C, we generate 6 response bits per each ISQ (i.e., 6 bits per 6 instructions) in the input challenge. Therefore, the number of the instructions needed for an $n$-bit response is: $\lceil n / 6\rceil \cdot 6$. For example, we will need an input challenge consisting of 132 instructions (or 22 ISQs) to generate an 128-bit response. Conversely, the existing fully software based processor PUF [43] (we refer to as soft-PUF) uses 12.8k instructions as challenge to generate the 128-bit response. Using the analysis phase of Figure 4 and the extracted VCD files (see Section 4), 


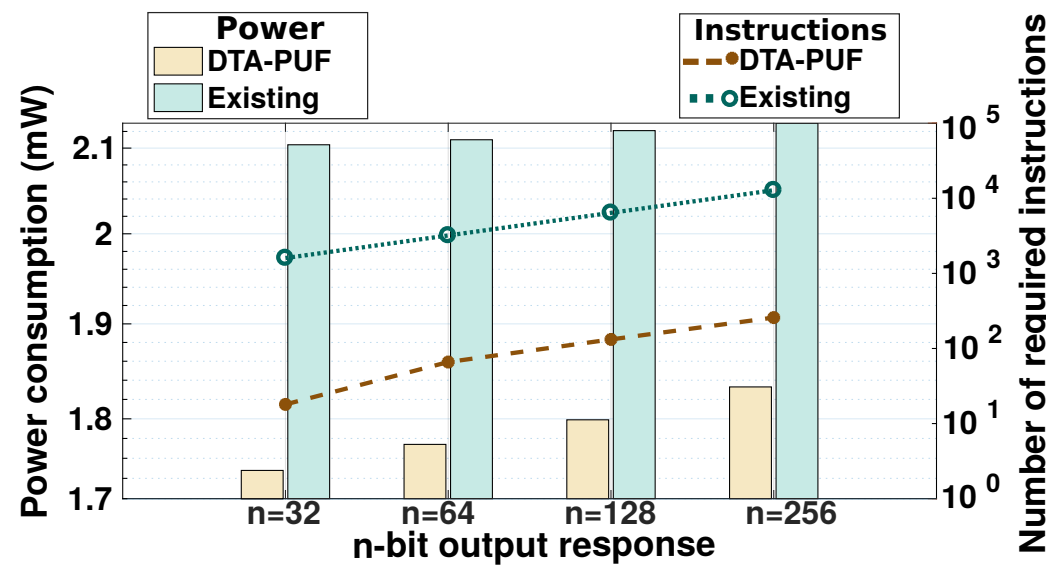

Fig. 10. Power consumption and required number of instructions across DTA-PUF and the existing software processor PUF [43] under different response bits.

we estimate the dynamic power consumption under different number of response bits. Figure 10 compares the power consumption incurred by our PUF with the one obtained by soft-PUF across increasing number of response bits. The number of the instructions that DTA-PUF and soft-PUF require for generating responses is also depicted in the right y axis of the same figure. Note that for the sake of this comparison, we simulate the target FPU using different number of instructions required for DTA-PUF and soft-PUF to generate the $n$-bit response. As shown in Figure 10, DTA-PUF requires up-to $50 \times$ less instructions than soft-PUF to generate output responses. Such a property, allows DTA-PUF to save up-to $26 \%$ power when compared with soft-PUF.

As we discussed, DTA-PUF interrupts normal microprocessor operation and uses the target design only as a PUF. This imposes an execution time penalty on the executed applications, which strongly depends on the number of instructions required to generate an n-bit PUF response. In fact, this penalty will be equal to the execution time of the PUF query procedure. To better understand the involved overhead, we define the execution time $(E x T)$ of any PUF running at $C l k P_{\text {red }}$ as:

$$
E x T=\# \text { cycles } \times C l k P_{\text {red }}=(\# \text { instructions }+ \text { pipeline_depth }) \times C l k P_{\text {red }}
$$

As explained, DTA-PUF requires 132 instructions to generate an 128-bit response leading to $E x T_{D T A-P U F}=138 \times C l k P_{\text {red }}$. When compared to the execution time overhead incurred by softPUF for generating the 128 -bit response $\left(E x T_{\text {soft-PUF }}=12806 \times C l k P_{\text {red }}\right)$, DTA-PUF reduces the execution time penalty by $\sim 92.8 \times$.

\subsection{Potential Use Cases}

As shown, DTA-PUF achieves a low power security primitive without needing any hardware addition. Such a PUF can be used in any (micro)processor to provide a secure challenge-response mechanism. Additional use cases of DTA-PUF are discussed next.

5.7.1 IoT Device Identification \& Authentication. Lightweight and low cost authentication and identification are very important security aspects for IoT devices because they often cannot afford resource-demanding cryptographic protocols. DTA-PUF is a fully software derived (i.e., no need for extra circuitry or design changes), low power solution to secure IoT. Further, DTA-PUF proposes a simple and fast challenge-response mechanism that generates relatively large output responses, while limiting the processing effort and size of input challenges. Therefore, DTA-PUF could be 
extremely beneficial for low power device ID and authentication in IoT nodes or in any other system that lacks computational resources [27].

5.7.2 DTA-PUF \& Approximate Computing. DTA-PUF leverages the inherent complex manifestation of dynamic timing errors to provide a lightweight secure protocol. In our design, timing errors are induced by overclocking the design under test. Increasing the clock frequency (i.e., reducing the clock period) is a common technique that the approximate computing paradigm $[45,71]$ exploits to significantly reduce the energy consumption of a system. Frequency scaling may increase the energy savings, but as we showcase in this paper, it comes with the cost of timing errors. The quality loss incurred by these errors $[38,62,64]$ may be tolerated by inherent resilient algorithms such as signal/image processing, machine learning and scientific computation [23, 30]. Hence, DTA-PUF could be jointly considered with approximate computing methods to offer resource and power savings while sacrificing quality in applications that are amenable to approximations and can tolerate inaccurate results. In such a system, the PUF responses could be extracted from the error prone least significant bits of approximate computations. As shown in Section 5.4, the bits in which error can be tolerated in approximate calculations are also the bits from which the best PUF response can be extracted. A scheme making proper use of this principle could in effect generate PUF responses while incurring near zero power and execution time costs relative to normal operation.

Finally, modern platforms allow operations beyond the nominal voltage/frequency values for improving energy efficiency $[9,47,48]$. This makes it possible to integrate DTA-PUF in these platforms without requiring any extra mechanism (e.g., clock generator, voltage regulator) to dynamically adjust the voltage or frequency margins.

\subsection{Discussion on DTA-PUF Vulnerability to Attacks}

The proposed PUF leverages the data-dependent dynamic timing behaviour of pipelined cores to generate unique and reliable responses. At the most fundamental level, the entropy source is the variance in delays within the core. It has been previously demonstrated that many delay based PUFs are vulnerable to attacks based on ML modelling [53]. Further, obfuscation of the PUF response and other established methods of impeding ML modelling attacks are not possible without the addition of hardware, which invalidates the fully software based nature of the design. As such, while a full analysis of ML attacks against the proposed design is beyond the scope of this work (though it may form the basis of future work), it is useful to discuss the possible vulnerability to this kind of attack.

Conventional delay based PUFs are in most cases fairly simplistic in their underlying architecture. In the case of conventional arbiter and RO PUFs this can be as simple as a fixed size chain of delay elements $[29,60]$. Derivation of a mathematical model able to represent the variables which contribute to entropy within them is fairly trivial. Conversely, the scale and complexity of even a moderately complex pipelined core is an order of magnitude higher in regard to the number of variables and their interaction to form the final PUF response. As such, it is not clear that deriving a model will be trivial in comparison to conventional delay based PUFs, even with a sufficiently large set of training data.

In regards to the gathering of training CRPs, there are also non-trivial challenges. Unlike in conventional delay based PUFs, the possible inputs and the challenge set significantly vary. In fact, depending on the exact implementation, the challenge set may be as small as 15K ISQs out of the total possible $\left(6 \times 2^{64} \times 2^{64}\right)^{6} \approx 7.24 \times 10^{235}$ (based on 6 instruction ISQs with two 64 bit operands as discussed in section 5.A). The vast majority of the ISQs outside of this set will produce minimal or zero entropy (since they do not produce timing errors) and hence will not be useful for training a model. It has been estimated [67] that roughly $99 \%$ of timing critical paths are triggered by less 
than $10 \%$ of all ISQs; and the probability to obtain the worst-case input conditions, which result in timing errors, is extremely small [21].

Which ISQs are useful for triggering critical paths can be determined quite readily with full access to the original design files and using the methods outlined above. Nevertheless, for an attacker without such access or prior knowledge of the ISQs, to target the model must be trained using arbitrarily chosen ISQs. As the vast majority of these will be non-entropic, this can be compared to the well known poisoning attack [36].

Hence, even if an adversary has full access to the PUF for a relatively long period the odds of generating a valid training dataset without prior knowledge of the challenge set, are very low. In fact, an adversary need to know precise details of the chip as well as all the parameters that affect the dynamic timing behaviour of errors (i.e., clock reduction, type of instructions, input operands, instruction execution history). In effect, the necessity of acquiring or deriving the challenge set itself acts as a layer of obfuscation. However, the security of DTA-PUF would be compromised if an attacker had access to the challenge set and could overwrite it. To protect challenges from being overwritten, secure access mechanisms and data authentication protocols (e.g., digital signature and cryptographic hash) have been proposed [57, 59]. Note that we focus on the scenario that the input challenge set is secured and cannot be used for malicious activities.

While neither of these factors in themselves render the proposed PUF definitively immune to modelling attacks [25], they are non-trivial barriers. This raises the bar of entry and forces an adversary to expend greater time and resources than it would seem at first glance.

\section{RELATED WORK}

In this section, we present an overview of work in the field to date regarding processor based PUFs, considering designs which are non-software based, requiring the addition of substantial additional hardware; lightweight non-software based, where the additional hardware required is relatively small; and fully software based processor PUFs where the response is entirely derived using software. The comparison of all the PUFs explained below is shown in Table 1.

\subsection{Fully Software Processor Based PUFs}

There are relatively few designs for processor derived PUFs which are fully software based (i.e., which require no additional hardware whatsoever to perform the PUF query procedure).

Maiti et al. [43] propose a fully software processor based PUF. The principle of operation is that by altering the clock frequency of a processor by a specific amount and running a sequence of instructions some large number of times, the failure rate is unique to a given processor at a given frequency. This is because for a given instruction there is a curve of increasing failure rate approaching $100 \%$ as frequency increases with the start and end points of this curve determined by low level variances unique to a given processor. So, at a clock reduction of, for example, $26 \%$ for the same instruction set no failures may occur on one chip, $20 \%$ failure rate on another, $76 \%$ faillure rate on a third etc., depending on the position and shape of the failure curves in regards to increasing frequency for each chip. The failure rate is encoded such that minimal failures constitute a binary 00 , the lower half of the curve 01 , the upper half 10 , and near or complete failure 11 .

This has the advantage of being a fully software derived design, assuming that a clock phaselocked loop (PLL) or other programmable clock control mechanism is available. However, it has several drawbacks. The design as proposed is highly, though not ideally, reliable but the performance in terms of inter-chip uniqueness is far from ideal. A given instruction and operand can begin to fail anywhere within a quite wide range of frequencies, meaning that multiple frequency values must be used to ensure uniqueness. Due to this, the total available response bits is also somewhat limited by the granularity of control over the frequency. Characterising this PUF is challenging as every

, Vol. -, No. -, Article -. Publication date: - 
instruction must be characterised at every frequency within the given range to gather full CRP data. Further, the generation of every two response bits requires the execution of some large number of instructions in the order of hundreds or thousands. Lastly, the source of entropy is the number of failed instructions at a given point, but this ignores the fact that two failed instructions with non-correctable outputs may in fact have differing levels of bitwise error and different positioning of errors within the output of nominally "failed" instructions.

\subsection{Lightweight Non-Software Processor Based PUFs}

Several processor derived PUFs have been proposed which attempt to minimise the hardware overhead if additional circuitry is needed, or which are nominally software based but make use of test structures that may not be present in the right configuration in practice.

PUFatt, proposed by Kong et al. [40] is comparatively lightweight. It inserts delay arbiters after redundant (i.e., identical) ALU circuits within a processor design. This minimises the hardware resources required as only the arbiter components themselves are new. The same instruction is passed to each ALU and the response bits generated based on the relative speed of execution. The execution time is a product of the path delay variances within each ALU. This is very similar conceptually to well studied delay based PUFs, such as the arbiter PUF [29], in that the entropy source is a product of cumulative path delay variances. The resultant PUF response is not ideal but is within acceptable bounds with $35.9 \%$ uniqueness on average and average reliability of $88.7 \%$. An obfuscation scheme is proposed which increases the measured uniqueness to $44.6 \%$ at the cost of requiring additional post-processing.

While this design minimises the extra hardware resource usage, it is still not fully software based. Performance in regard to security metrics is passable but not ideal and much lower in certain metrics than other processor PUF designs such as that proposed by Maiti et al. [43]. In addition, the reliability of the design is measured only for the raw output and not for the final output of the proposed obfuscation scheme, which, by the admission of the authors, can produce distinctly different outputs if even a small number of input bits (from the raw PUF response) are incorrect. As this design is conceptually similar to many well studied delay PUFs and does not implement any kind of CRP obfuscation, it may be vulnerable to common ML modelling attacks [25].

Kong and Koushanfar [39] take a similar approach, implementing arbiters after redundant ALU components. This work is more developed and applies some of the obfuscation techniques now common to delay PUF architectures, which at least partially mitigates the risk of ML modelling attacks. As with PUFatt [40], this design is not fully software based. It performs fairly well with a uniqueness in the order of $38 \%$ and the authors propose a targeted aging algorithm which can reportedly increase this to $45 \%$. However, it is pointed out by the authors that this algorithm could also be used maliciously to reduce the uniqueness of the PUF, and thus rendering it more vulnerable to modelling. Preventing such an attack would require additional aging detection circuitry in addition to the arbiters needed for PUF operation.

Scan PUF, proposed by Zheng et al. [69], uses the delay variances in scan chains, a common design-for-test structure, as the entropy source. Such structures are not universally present in commodity devices but are very common and as such this design is largely software based. However, additional circuitry is required in order to extract responses from the scan chain structures. This design performs well in key security metrics with a uniqueness of $47 \%$ and reliability above $95 \%$ at room temperature in practical tests.

Whilst the scan chains are already present on the device, it is necessary to generate precise input signals in order to generate a PUF response from them. In Scan PUF, this is done by adding a small amount of signal generation circuitry. This prevents the design from being truly software based but has significantly lower hardware resource overheads than many of the other proposed designs. It is

, Vol. -, No. -, Article -. Publication date: -. 
Table 1. Processor Derived PUF designs

\begin{tabular}{|c|c|c|c|c|c|}
\hline PUF & Type & $\begin{array}{l}\text { Hardware } \\
\text { Additions }\end{array}$ & $\begin{array}{c}\text { Practical } \\
\text { Considerations }\end{array}$ & $\begin{array}{l}\text { Average } \\
\text { Uniqueness }\end{array}$ & $\begin{array}{l}\text { Suitable for } \\
\text { Resource } \\
\text { Constrained } \\
\text { Devices }\end{array}$ \\
\hline $\begin{array}{c}\text { HELP } \\
{[5]}\end{array}$ & Non-Software & Large test circuit & $\begin{array}{l}\text { Long query time, } \\
\text { Limited response size }\end{array}$ & $49.9 \%$ & $x$ \\
\hline $\begin{array}{c}\text { PASC } \\
{[8]}\end{array}$ & Non-Software & Instruction logic & $\begin{array}{l}\text { Must implement a } \\
\text { custom instruction }\end{array}$ & $38.2 / 49.2 \%$ & $x$ \\
\hline $\begin{array}{l}\text { Maiti et al. } \\
\text { [43] }\end{array}$ & Fully Software & None & $\begin{array}{l}\text { Difficult to characterise, } \\
\text { Programmable PLL/clock } \\
\text { generator required, } \\
\text { High power consumption }\end{array}$ & $37.5 \%$ & $x$ \\
\hline $\begin{array}{l}\text { PUFAtt } \\
{[40]}\end{array}$ & $\begin{array}{l}\text { Lightweight } \\
\text { Non-Software }\end{array}$ & $\begin{array}{l}\text { Arbiters after } \\
\text { redundant ALU } \\
\text { components }\end{array}$ & $\begin{array}{l}\text { A means to synchronise } \\
\text { ALU inputs is required }\end{array}$ & $35.9 \%$ & $x$ \\
\hline $\begin{array}{c}\text { DScan PUF } \\
\text { [72] }\end{array}$ & $\begin{array}{l}\text { Lightweight } \\
\text { Non-Software }\end{array}$ & $\begin{array}{c}\text { Scan chains, } \\
\text { Additional control } \\
\text { circuitry }\end{array}$ & $\begin{array}{l}\text { Response generation } \\
\text { method is highly complex, } \\
\text { Programmable PLL/clock } \\
\text { generator required }\end{array}$ & $49.9 \%$ & $x$ \\
\hline $\begin{array}{c}\text { Scan PUF } \\
{[69]}\end{array}$ & $\begin{array}{l}\text { Lightweight } \\
\text { Non-Software }\end{array}$ & $\begin{array}{c}\text { Scan chains, } \\
\text { Modifications to } \\
\text { signal generating } \\
\text { circuits }\end{array}$ & $\begin{array}{l}\text { Requires generation of } \\
\text { controlled input signals }\end{array}$ & $47 \%$ & $x$ \\
\hline DTA-PUF & Fully Software & None & $\begin{array}{l}\text { Single raised clock } \\
\text { domain is required }\end{array}$ & $31.4 \%$ & $\checkmark$ \\
\hline
\end{tabular}

also noted by the authors that the implementation of Scan PUF produces small increases in power consumption in test modes. No analysis is given of the power overheads of switching between test and functional mode to access the PUF response.

Zheng et al. [72] later proposed DScan PUF. This design operates on similar principles to the earlier Scan PUF [69] but employs a novel control circuit on top of the already present scan chains. This control circuit facilitates the design of a PUF with more robust characteristics than Scan PUF. The resultant PUF is ideal in terms of uniqueness and can generate highly reliable responses. However, as with the Scan PUF, while it is relatively low overhead in terms of hardware resources, it is not truly software based as design changes and some hardware resource usage are required to implement it.

\subsection{Non-Software Processor Based PUFs}

Aarestad et al. proposed HELP (Hardware Embedded Delay PUF) [5], a design which makes use of an added on-chip test structure called "REBEL" to measure the path delays directly. Several extraction methodologies are proposed to convert pairs of path delays into a binary PUF response. The rate of generation of response bits is limited, but the response that can be generated is both highly unique and reliable under the proposed conditions. 
The auxiliary test circuit used in HELP requires a large amount of hardware resource - more than $100 \%$ of the size of the circuit being measured in the experiments reported. Notably, query time is very long in comparison to what is typical in PUF designs with a rate of less than 3 bits per second on the test hardware. Furthermore, the methods of key extraction are computationally intensive and limit the maximum number of bits which can be generated to a practical upper bound of 256 for the most robust generation method. This design excellently performs in regards to security metrics, but the hardware costs in addition to the computational cost and corresponding power cost renders it unsuitable for resource constrained systems.

Aysu and Schaumont proposed PASC [8] to address some of the weaknesses in the work of Maiti et al. [43]. Specifically they note the requirement for the ability to control the clock frequency over quite a large range in order to target the failure points of certain instructions. They propose that it is not practical to use the native instructions for this reason. To this end, PASC [8] employs a custom instruction designed to fail at around the same frequency in all devices. The PUF responses are differentiated by the slope of the failure curve, rather than both slope and positioning on the frequency spectrum.

This has several advantages. Only a single raised clock domain is required to implement the PUF and the output is both reasonably unique and highly reliable. Nevertheless, outside of the domain of FPGAs the implementation of the required custom instruction requires architectural changes to the processor and is thus non-software. Further, the problem of needing multiple clock domains only applies if the failure rate is used as the entropy source.

Overall, in this paper, we aim at implementing a fully software, processor based PUF design that combines the positive aspects of the PUFs discussed above and addresses their limitations. As has been demonstrated, DTA-PUF extracts entropy from instructions at a single raised clock domain by proper selection of instruction sequences and by looking at the error rate within the failed instructions. This allow us to provide a simple and quick challenge-response mechanism that generates output bits with a considerably small number of input instructions. Such properties render DTA-PUF extremely suitable for low power, resource-scarce devices though the average uniqueness value of our PUF deviates from the ideal value of $50 \%$.

\section{CONCLUSIONS}

In this paper, we presented DTA-PUF, a fully software derived PUF design which is based on the existing timing variability of pipelined cores. Our novel method closely intertwines the PUF with the underlying circuit, exploiting the inherent complexity of timing error occurrence. Our evaluation results suggest that the proposed PUF shows a good amount of uniqueness and reliability in terms of the PUF responses and, at the same time, incurs limited or no processing costs and minimal power overheads. Moreover, the high bit generation speed of our PUF results in significant power savings when compared with existing fully software based processor PUFs. DTA-PUF can be used as a lightweight mechanism to address emerging challenges in hardware security of devices that have power and computational resource concerns, such as IoT nodes, especially in regard to device identification and authentication.

\section{ACKNOWLEDGMENTS}

The presented research effort is partially supported by the UK Engineering and Physical Science Research Council (EPSRC) under grant EP/N508664/-CSIT2.

\section{REFERENCES}

[1] IEEE 754-2008. IEEE 754-2008 Standard for Floating-Point Arithmetic..

[2] NanGate FreePDK45 Open Cell Library, http://nangate.com. 
[3] Online available at: https://www.idc.com/. International Data Corporation.

[4] OpenRISC Community, "OpenRISC 1000 architecture manual.".

[5] J. Aarestad, P. Ortiz, D. Acharyya, and J. Plusquellic. 2013. HELP: A Hardware-Embedded Delay PUF. IEEE Design \& Test 30, 2 (2013), 17-25.

[6] M. N. Alraja, M. M. J. Farooque, and B. Khashab. 2019. The Effect of Security, Privacy, Familiarity, and Trust on Users Attitudes Toward the Use of the IoT-Based Healthcare: The Mediation Role of Risk Perception. IEEE Access 7 (2019).

[7] H. Amrouch, B. Khaleghi, A. Gerstlauer, and J. Henkel. 2017. Towards aging-induced approximations. In DAC. 1-6.

[8] A. Aysu and P. Schaumont. 2013. PASC: Physically authenticated stable-clocked soc platform on low-cost FPGAs. In 2013 International Conference on Reconfigurable Computing and FPGAs (ReConFig). 1-6.

[9] Anys Bacha and Radu Teodorescu. 2014. Using ECC Feedback to Guide Voltage Speculation in Low-Voltage Processors. In Proceedings of the 47th Annual IEEE/ACM International Symposium on Microarchitecture (Cambridge, United Kingdom) (MICRO-47). IEEE Computer Society, USA, 306-318. https://doi.org/10.1109/MICRO.2014.54

[10] B. M. S. Bahar Talukder, B. Ray, D. Forte, and M. T. Rahman. 2019. PreLatPUF: Exploiting DRAM Latency Variations for Generating Robust Device Signatures. IEEE Access 7 (2019), 81106-81120.

[11] D. H. Bailey, E. Barszcz, J. T. Barton, D. S. Browning, R. L. Carter, L. Dagum, R. A. Fatoohi, P. O. Frederickson, T. A. Lasinski, R. S. Schreiber, H. D. Simon, V. Venkatakrishnan, and S. K. Weeratunga. 1991. The NAS parallel benchmarks summary and preliminary results. In Supercomputing. 158-165.

[12] G. Bakirtzis, B. J. Simon, A. G. Collins, C. H. Fleming, and C. R. Elks. 2019. Data-Driven Vulnerability Exploration for Design Phase System Analysis. IEEE Systems fournal (2019), 1-10.

[13] J. Bhasker and R. Chadha. New York, USA: Springer, 2009.. Static Timing Analysis for Nanometer Designs: A Practical Approach.

[14] K. A. Bowman, J. W. Tschanz, S. L. Lu, P. A. Aseron, M. M. Khellah, A. Raychowdhury, B. M. Geuskens, C. Tokunaga, C. B. Wilkerson, T. Karnik, and V. K. De. 2011. A $45 \mathrm{~nm}$ Resilient Microprocessor Core for Dynamic Variation Tolerance. IEEE Journal of Solid-State Circuits 46, 1 (2011), 194-208.

[15] Chun-Kai Chang, Wenqi Yin, and Mattan Erez. 2019. Assessing the impact of timing errors on HPC applications. In Proceedings of the International Conference for High Performance Computing, Networking, Storage and Analysis, SC 2019, Denver, Colorado, USA, November 17-19, 2019, Michela Taufer, Pavan Balaji, and Antonio J. Peña (Eds.). ACM, 70:1-70:19. https://doi.org/10.1145/3295500.3356184

[16] B. Cline, K. Chopra, D. Blaauw, and Y. Cao. 2006. Analysis and Modeling of CD Variation for Statistical Static Timing. In 2006 IEEE/ACM International Conference on Computer Aided Design. 60-66.

[17] Jeremy Constantin, Andreas Peter Burg, Zheng Wang, Anupam Chattopadhyay, and Georgios Karakonstantis. 2016. Statistical fault injection for impact-evaluation of timing errors on application performance. In DAC. 13:1-13:6. https://doi.org/10.1145/2897937.2898095

[18] Abhishek Das, Serkan Ozdemir, Gokhan Memik, Joseph Zambreno, and Alok Choudhary. 2007. Mitigating the Effects of Process Variations : Architectural Approaches for Improving Batch Performance.

[19] J. Delvaux and I. Verbauwhede. 2013. Side channel modeling attacks on 65nm arbiter PUFs exploiting CMOS device noise. In 2013 IEEE International Symposium on Hardware-Oriented Security and Trust (HOST). 137-142.

[20] S. Dighe, S. R. Vangal, P. Aseron, S. Kumar, T. Jacob, K. A. Bowman, J. Howard, J. Tschanz, V. Erraguntla, N. Borkar, V. K. De, and S. Borkar. 2011. Within-Die Variation-Aware Dynamic-Voltage-Frequency-Scaling With Optimal Core Allocation and Thread Hopping for the 80-Core TeraFLOPS Processor. IEEE fournal of Solid-State Circuits 46, 1 (2011), 184-193.

[21] R. G. Dreslinski, M. Wieckowski, D. Blaauw, D. Sylvester, and T. Mudge. 2010. Near-Threshold Computing: Reclaiming Moore's Law Through Energy Efficient Integrated Circuits. Proc. IEEE 98, 2 (2010), 253-266.

[22] Anh Nguyen Duc, Ronald Jabangwe, Pangkaj Paul, and Pekka Abrahamsson. 2017. Security Challenges in IoT Development: A Software Engineering Perspective. In Proceedings of the XP2017 Scientific Workshops (Cologne, Germany) (XP '17). Association for Computing Machinery, New York, NY, USA, Article 11, 5 pages. https://doi.org/10.1145/ 3120459.3120471

[23] Hadi Esmaeilzadeh, Adrian Sampson, Luis Ceze, and Doug Burger. 2012. Architecture support for disciplined approximate programming. In Proceedings of the 17th International Conference on Architectural Support for Programming Languages and Operating Systems, ASPLOS 2012, London, UK, March 3-7, 2012, Tim Harris and Michael L. Scott (Eds.) ACM, 301-312. https://doi.org/10.1145/2150976.2151008

[24] W. Yan F. Tehranipoor, N. Karimian and J. A. Chandy. 2017. DRAM-Based Intrinsic Physically Unclonable Functions for System-Level Security and Authentication. IEEE TVLSI 25, 3 (2017), 1085-1097.

[25] Fatemeh Ganji, Shahin Tajik, Fabian Fäßler, and Jean-Pierre Seifert. 2016. Strong Machine Learning Attack Against PUFs with No Mathematical Model. In CHES (Lecture Notes in Computer Science, Vol. 9813). Springer, 391-411.

[26] Fatemeh Ganji, Shahin Tajik, and Jean-Pierre Seifert. 2016. PAC learning of arbiter PUFs. F. Cryptogr. Eng. 6, 3 (2016), 249-258. https://doi.org/10.1007/s13389-016-0119-4 
[27] M. Gao, Q. Wang, M. T. Arafin, Y. Lyu, and G. Qu. 2017. Approximate computing for low power and security in the Internet of Things. Computer 50, 6 (2017), 27-34.

[28] D. Garyfallou, I. Tsiokanos, N. Evmorfopoulos, G. Stamoulis, and G. Karakonstantis. 2020. Accurate Estimation of Dynamic Timing Slacks using Event-Driven Simulation. In 2020 21st International Symposium on Quality Electronic Design (ISQED). 225-230. https://doi.org/10.1109/ISQED48828.2020.9137017

[29] B. Gassend, D. Clarke, M. van Dijk, and S. Devadas. 2002. Silicon physical random functions. In CCS. 148.

[30] Beayna Grigorian, Nazanin Farahpour, and Glenn Reinman. 2015. BRAINIAC: Bringing reliable accuracy into neurallyimplemented approximate computing. In 21st IEEE International Symposium on High Performance Computer Architecture, HPCA 2015, Burlingame, CA, USA, February 7-11, 2015. IEEE Computer Society, 615-626. https://doi.org/10.1109/HPCA. 2015.7056067

[31] Puneet Gupta, Yuvraj Agarwal, Lara Dolecek, Nikil D. Dutt, Rajesh K. Gupta, Rakesh Kumar, Subhasish Mitra, Alexandru Nicolau, Tajana Simunic Rosing, Mani B. Srivastava, Steven Swanson, and Dennis Sylvester. 2013. Underdesigned and Opportunistic Computing in Presence of Hardware Variability. TCAD (2013).

[32] B. Halak, M. Zwolinski, and M. S. Mispan. 2016. Overview of PUF-based hardware security solutions for the internet of things. In 2016 IEEE 59th International Midwest Symposium on Circuits and Systems (MWSCAS). 1-4.

[33] T. Hiramoto, K. Takeuchi, T. Mizutani, A. Ueda, T. Saraya, M. Kobayashi, Y. Yamamoto, H. Makiyama, T. Yamashita, H. Oda, S. Kamohara, N. Sugii, and Y. Yamaguchi. 2016. Ultra-low power and ultra-low voltage devices and circuits for IoT applications. In 2016 IEEE Silicon Nanoelectronics Workshop (SNW). 146-147.

[34] Giang Hoang, Robby Bruce Findler, and Russ Joseph. 2011. Exploring circuit timing-aware language and compilation. In ASPLOS. ACM, 345-356.

[35] D. E. Holcomb, W. P. Burleson, and K. Fu. 2009. Power-Up SRAM State as an Identifying Fingerprint and Source of True Random Numbers. IEEE Trans. Computers (Sep. 2009). https://doi.org/10.1109/TC.2008.212

[36] M. Jagielski, A. Oprea, B. Biggio, C. Liu, C. Nita-Rotaru, and B. Li. 2018. Manipulating Machine Learning: Poisoning Attacks and Countermeasures for Regression Learning. In 2018 IEEE Symposium on Security and Privacy (SP). 19-35.

[37] X. Jiao, A. Rahimi, Y. Jiang, J. Wang, H. Fatemi, J. P. de Gyvez, and R. K. Gupta. 2018. CLIM: A Cross-Level WorkloadAware Timing Error Prediction Model for Functional Units. IEEE Trans. Computers (2018). https://doi.org/10.1109/TC. 2017.2783333

[38] Georgios Karakonstantis, Abhijit Chatterjee, and Kaushik Roy. 2011. Containing the Nanometer "Pandora-Box": Cross-Layer Design Techniques for Variation Aware Low Power Systems. IEEE 7. Emerg. Sel. Topics Circuits Syst. 1, 1 (2011), 19-29. https://doi.org/10.1109/JETCAS.2011.2135590

[39] J. Kong and F. Koushanfar. 2014. Processor-Based Strong Physical Unclonable Functions With Aging-Based Response Tuning. IEEE TETC (March 2014).

[40] J. Kong, F. Koushanfar, P. K. Pendyala, A. Sadeghi, and C. Wachsmann. 2014. PUFatt: Embedded platform attestation based on novel processor-based PUFs. In DAC. 1-6. https://doi.org/10.1145/2593069.2593192

[41] N. Li, D. Liu, and S. Nepal. 2017. Lightweight Mutual Authentication for IoT and Its Applications. IEEE Transactions on Sustainable Computing 2 (2017).

[42] A. Maiti and P. Schaumont. 2011. Improved Ring oscillator PUF: An FPGA-friendly secure primitive. fournal of Cryptology 24 (2011), 375-397.

[43] A. Maiti and P. Schaumont. 2012. A novel microprocessor-intrinsic Physical Unclonable Function. In FPL. $380-387$. https://doi.org/10.1109/FPL.2012.6339208

[44] M. S. Mispan, H. Su, M. Zwolinski, and B. Halak. 2018. Cost-efficient design for modeling attacks resistant PUFs. In 2018 Design, Automation Test in Europe Conference Exhibition (DATE). 467-472.

[45] Sparsh Mittal. 2016. A Survey of Techniques for Approximate Computing. ACM Comput. Surv. 48, 4 (2016), $62: 1-62: 33$. https://doi.org/10.1145/2893356

[46] Lev Mukhanov, Dimitrios S. Nikolopoulos, and Bronis R. de Supinski. 2015. ALEA: Fine-Grain Energy Profiling with Basic Block Sampling. In 2015 International Conference on Parallel Architecture and Compilation, PACT 2015, San Francisco, CA, USA, October 18-21, 2015. IEEE Computer Society, 87-98. https://doi.org/10.1109/PACT.2015.16

[47] G. Papadimitriou, M. Kaliorakis, A. Chatzidimitriou, D. Gizopoulos, P. Lawthers, and S. Das. 2017. Harnessing Voltage Margins for Energy Efficiency in Multicore CPUs. In 2017 50th Annual IEEE/ACM International Symposium on Microarchitecture (MICRO). 503-516.

[48] K. Parasyris, P. Koutsovasilis, V. Vassiliadis, C. D. Antonopoulos, N. Bellas, and S. Lalis. 2018. A Framework for Evaluating Software on Reduced Margins Hardware. In 2018 48th Annual IEEE/IFIP International Conference on Dependable Systems and Networks (DSN). 330-337. https://doi.org/10.1109/DSN.2018.00043

[49] Taejoon Park and Kang G. Shin. 2004. LiSP: A Lightweight Security Protocol for Wireless Sensor Networks. ACM Trans. Embed. Comput. Syst. 3, 3 (Aug. 2004), 634-660. https://doi.org/10.1145/1015047.1015056

[50] Md. Tauhidur Rahman, Alison Hosey, Zimu Guo, Jackson Carroll, Domenic Forte, and Mark Tehranipoor. 2017. Systematic Correlation and Cell Neighborhood Analysis of SRAM PUF for Robust and Unique Key Generation. $\mathcal{F}$.

, Vol. -, No. -, Article -. Publication date: -. 
Hardware and Systems Security 1, 2 (2017), 137-155. https://doi.org/10.1007/s41635-017-0012-3

[51] A. Raychowdhury, S. Ghosh, and K. Roy. 2005. A novel on-chip delay measurement hardware for efficient speed-binning. In 11th IEEE International On-Line Testing Symposium. 287-292.

[52] J. N. Rodrigues, M. Kamuf, H. Hedberg, and V. Owall. 2005. A manual on ASIC front to back end design flow. In 2005 IEEE International Conference on Microelectronic Systems Education (MSE'05). 75-76.

[53] Ulrich Rührmair, Frank Sehnke, Jan Sölter, Gideon Dror, Srinivas Devadas, and Jürgen Schmidhuber. 2010. Modeling attacks on physical unclonable functions. In CCS. ACM, 237-249. https://doi.org/10.1145/1866307.1866335

[54] Ulrich Rührmair, Xiaolin Xu, Jan Sölter, Ahmed Mahmoud, Mehrdad Majzoobi, Farinaz Koushanfar, and Wayne Burleson. 2014. Efficient Power and Timing Side Channels for Physical Unclonable Functions. In Proceedings of the 16th International Workshop on Cryptographic Hardware and Embedded Systems - CHES 2014 - Volume 8731. Springer-Verlag, Berlin, Heidelberg, 476-492. https://doi.org/10.1007/978-3-662-44709-3_26

[55] S. Sakib, A. Milenković, M. T. Rahman, and B. Ray. 2020. An Aging-Resistant NAND Flash Memory Physical Unclonable Function. IEEE Transactions on Electron Devices 67, 3 (2020), 937-943.

[56] J. Sartori, A. Pant, R. Kumar, and P. Gupta. 2010. Variation-aware speed binning of multi-core processors. In 2010 11th International Symposium on Quality Electronic Design (ISQED). 307-314.

[57] M. Sauer, P. Raiola, L. Feiten, B. Becker, U. Rührmair, and I. Polian. 2017. Sensitized path PUF: A lightweight embedded physical unclonable function. In DATE. 680-685. https://doi.org/10.23919/DATE.2017.7927076

[58] Sophiane Senni, Lionel Torres, Gilles Sassatelli, and Abdoulaye Gamatie. 2016. Non-Volatile Processor Based on MRAM for Ultra-Low-Power IoT Devices. f. Emerg. Technol. Comput. Syst. 13, 2, Article 17 (Dec. 2016), 23 pages. https://doi.org/10.1145/3001936

[59] Dimitrios N. Serpanos and Artemios G. Voyiatzis. 2013. Security Challenges in Embedded Systems. ACM Trans. Embed. Comput. Syst. 12, 1s, Article 66 (March 2013), 10 pages. https://doi.org/10.1145/2435227.2435262

[60] G. E. Suh and S. Devadas. 2007. Physical Unclonable Functions for Device Authentication and Secret Key Generation. In DAC. 9-14.

[61] Ioannis Tsiokanos, Lev Mukhanov, Giorgis Georgakoudis, Dimitrios S. Nikolopoulos, and Georgios Karakonstantis. 2020. DEFCON: Generating and Detecting Failure-prone Instruction Sequences via Stochastic Search. In DATE. IEEE, 1121-1126.

[62] I. Tsiokanos, L. Mukhanov, and G. Karakonstantis. 2019. Low-Power Variation-Aware Cores based on Dynamic Data-Dependent Bitwidth Truncation. In 2019 Design, Automation Test in Europe Conference Exhibition (DATE). 698-703. https://doi.org/10.23919/DATE.2019.8714942

[63] Ioannis Tsiokanos, Lev Mukhanov, Dimitrios S. Nikolopoulos, and Georgios Karakonstantis. 2018. Variation-Aware Pipelined Cores through Path Shaping and Dynamic Cycle Adjustment: Case Study on a Floating-Point Unit. In Proceedings of the International Symposium on Low Power Electronics and Design, ISLPED 2018, Seattle, WA, USA, fuly 23-25, 2018. ACM, 52:1-52:6. https://doi.org/10.1145/3218603.3218617

[64] I. Tsiokanos, L. Mukhanov, D. S. Nikolopoulos, and G. Karakonstantis. 2019. Significance-Driven Data Truncation for Preventing Timing Failures. IEEE TDMR 19, 1 (March 2019), 25-36. https://doi.org/10.1109/TDMR.2019.2898949

[65] E. I. Vatajelu, G. D. Natale, M. S. Mispan, and B. Halak. 2019. On the Encryption of the Challenge in Physically Unclonable Functions. In 2019 IEEE 25th International Symposium on On-Line Testing and Robust System Design (IOLTS). $115-120$

[66] I. Verbauwhede, D. Mukhopadhyay, and S. S. Roy. 2016. Embedded Security. In 2016 29th International Conference on VLSI Design and 2016 15th International Conference on Embedded Systems (VLSID). 23-23.

[67] J. Xin and R. Joseph. 2011. Identifying and predicting timing-critical instructions to boost timing speculation. In 2011 44th Annual IEEE/ACM International Symposium on Microarchitecture (MICRO). 128-139.

[68] Yan Pan, Joonho Kong, S. Ozdemir, G. Memik, and Sung Woo Chung. 2009. Selective wordline voltage boosting for caches to manage yield under process variations. In 2009 46th ACM/IEEE Design Automation Conference. 57-62.

[69] Yu Zheng, A. R. Krishna, and S. Bhunia. 2013. ScanPUF: Robust ultralow-overhead PUF using scan chain. In 2013 18th Asia and South Pacific Design Automation Conference (ASP-DAC). 626-631.

[70] Rafael Zalman and Albrecht Mayer. 2014. A Secure but Still Safe and Low Cost Automotive Communication Technique. In Proceedings of the 51st Annual Design Automation Conference (San Francisco, CA, USA) (DAC '14). Association for Computing Machinery, New York, NY, USA, 1-5. https://doi.org/10.1145/2593069.2603850

[71] Hang Zhang, Mateja Putic, and John Lach. 2014. Low Power GPGPU Computation with Imprecise Hardware. In The 51st Annual Design Automation Conference 2014, DAC '14, San Francisco, CA, USA, fune 1-5, 2014. ACM, 99:1-99:6. https://doi.org/10.1145/2593069.2593156

[72] Y. Zheng, F. Zhang, and S. Bhunia. 2016. DScanPUF: A Delay-Based Physical Unclonable Function Built Into Scan Chain. IEEE Transactions on Very Large Scale Integration (VLSI) Systems 24, 3 (2016), 1059-1070. 